CERN LIBRARIES, GENEVA

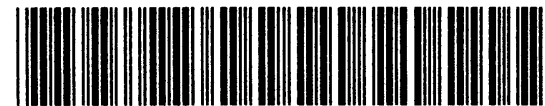

CM-P00057605
Anchives

Ref. TH. 806

\title{
ON A THEOREM BY CARRUTHERS
}

0. Steinmann

CERN - Geneva

Carruthers' theorem states that self-conjugate multiplets of bosons with half-integral isospin cannot exist. It is shown that this theorem can be derived from invariance under Iorentz transformations, CTP, and isospin rotations without using any other assumptions. 
In a recent paper Carruthers has proved that self-conjugate isospin multiplets of bosons with half-integral isospin cannot exist 1 ). The proof works with creation and annihilation operators, i.e., it makes use of the canonical commutation relations, as well as of some more complicated locality arguments. An alternate proof, based on essentially the same premises, has been given by Lee 2). Jin, on the other hand, derived this theorem from Wightman's axioms 3), making full use of the machinery of axiomatic field theory. In this note we wish to point out that the same result can be obtained from invariance considerations alone, without making use of locality, positivity of the energy, or the existence of the vacuum. More precisely, what is needed is invariance under : a) the connected Poincaré group, b) the CTP operation, and c) the isospin group.

Let $\varphi_{i}^{\tau}(p)$ be a self-conjugate isospin multiplet of fields with integral spin. The members of the multiplet are labelled by the value $\tau$ of $I_{3}$. The subscript $i$ stands for the set of indices of a tensor representation of the Lorentz group. Self-conjugation means that with $\varphi^{\tau}$ also its CTP conjugate is a member of the multiplet. Evidently, this conjugate field must be $\varphi^{-\tau}$. More exactly we postulate the connection

$$
(-1)^{5} \theta^{-1} \varphi_{i}^{\tau}(p) \theta \equiv \varphi_{i}^{\tau *}(-p)=\eta_{\tau}(p) \varphi_{i}^{-\tau}(p),
$$

where $\eta_{\tau}(p)$ is a Lorentz invariant function, $s$ is the spin of the fields involved, and $\theta$ is the CTP operator. That the connection between $\varphi^{\tau}$ and $\varphi^{-\tau}$ has to be local in $p$ space follows from momentum conservation. [The commutator of the momentum operator $\mathrm{P}_{\mu}$ with both sides of (1) has to be the same.] From this it follows also that $\eta_{\tau}$ cannot contain derivations. This fact is, however, inessential for our argument. Iorentz invariance demands that the spin components cannot be mixed up in the transition from $\varphi^{\tau}$ to $\varphi^{-\tau}$. 
From (1) we obtain by taking its adjoint:

$$
\varphi_{i}^{\tau}(-p)=\overline{\eta_{\tau}}(p) \varphi_{i}^{-\tau *}(p)
$$

Application of CTP to (1) yields

$$
\varphi_{i}^{\tau}(p)=\overline{\eta_{r}}(p) \varphi_{i}^{-\tau *}(-p)
$$

Comparison of (2) and (3) shows that

$$
\eta_{r}(-p)=\eta_{r}(p)
$$

We consider an isospin rotation by $180^{\circ}$ around the one axis represented by the unitary operator $\mathrm{R}$. The corresponding $\mathrm{SU}_{2}$ matrix is (io 1 . Thus,

$$
\begin{aligned}
& R^{-1} \varphi_{i}^{\tau}(p) R=i^{2 I} \varphi_{i}^{-\tau}(p), \\
& R^{-1} \varphi_{i}^{\tau^{*}}(p) R=i^{-2 I} \varphi_{i}^{-\tau^{*}}(p),
\end{aligned}
$$

where I is the isospin of the multiplet.

Repeated application of (1), (2), (5) yields; if (4) is taken into account:

$$
\begin{aligned}
\varphi_{i}^{-\tau}(p) & =i^{-2 I} R^{-1} \varphi_{i}^{\tau}(p) R \\
& =i^{-2 I} \overline{\eta_{\tau}}(p) R^{-1} \varphi_{i}^{-\tau^{*}}(-p) R \\
& =(-1)^{-2 I} \overline{\eta_{\tau}}(p) \varphi_{i}^{\tau *}(-p) \\
& =(-1)^{-2 I} \overline{\eta_{\tau}}(p) \eta_{\tau}(p) \varphi_{i}^{-\tau}(p),
\end{aligned}
$$


and thus,

$$
\left|\eta_{\tau}(p)\right|^{2}=(-1)^{2 I}
$$

This is only possible for integral I and thus the theorem is proved.

Let us briefly consider the case of half-integral spin.

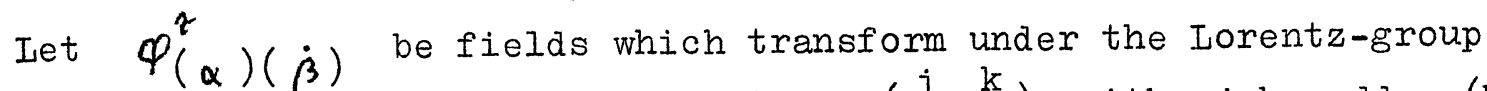
according to a representation of type $\left(\frac{j}{2}, \frac{k}{2}\right)$ with $j+k$ odd. (We use the notations of Ref. $\left.{ }^{4}\right)$ ). Together with these fields we have to consider a set of conjugate (in the spinor sense) fields $\varphi_{(\dot{\alpha})}^{\tau}(\beta)^{\text {. }}$ Equation (1) is replaced by

$$
\begin{aligned}
& \varphi_{(\alpha)(\dot{\beta})}^{\tau}(-p)=\eta_{\tau}(p) \varphi_{(\alpha)(\beta)}^{-\tau}(p) \\
& \varphi_{(\dot{\alpha})(\beta)}^{\tau}(-\beta)=\eta_{\tau}^{\prime}(p) \varphi_{(\alpha)(\dot{\beta})}^{-\tau}(p),
\end{aligned}
$$

where $\eta_{r}$ and $\eta_{r}^{\prime}$ need not be equal. This doubling of phase factors removes the contradiction. If we apply the same procedure as before, we obtain instead of (7) the condition

$$
\overline{\eta_{r}^{\prime}}(p) \eta_{\tau}(p)=(-1)^{2 I}
$$

which can be satisfied for any value of $I$. Indeed, the choice $\eta_{\tau}=$ $=i^{2 \tau}, \quad \eta_{\tau}^{\prime}=(-i)^{2 \tau}$ will satisfy all the conditions. 
1) P. Carruthers, Phys.Rev.Letters 18, 353 (1967).

2) H. Lee, Phys.Rev.Ietters 18, 1098 (1967).

3) Y.S. Jin, "A General Proof of Carruthers Theorem", Preprint NYO 2262 TA-148, Brown University (1967).

4) R.F. Streater and A.S. Wightman, "PCT, Spin and Statistics, and All That", Chapter 1 (Benjamin, New York), (1964). 